\title{
Speech Separation in Humans and Machines
}

Dan Ellis

Laboratory for Recognition and Organization of Speech and Audio Dept. Electrical Eng., Columbia Univ., NY USA

dpwe@ee.columbia.edu http://labrosa.ee.columbia.edu/

The Speech Separation Problem

2. Human Performance

3. Source Separation

4. Source Inference

5. Concluding Remarks 


\section{Speech Separation}

- Speech rarely occurs in isolation

- .. but recognizing mixed speech is a problem

0 .. for humans and machines

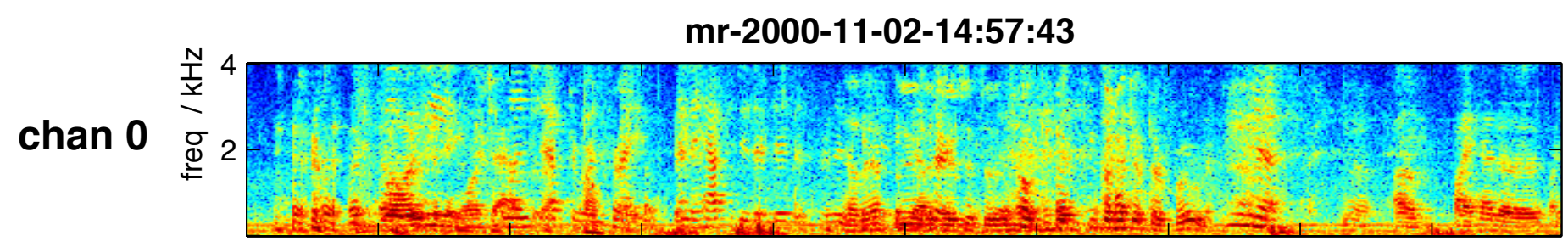

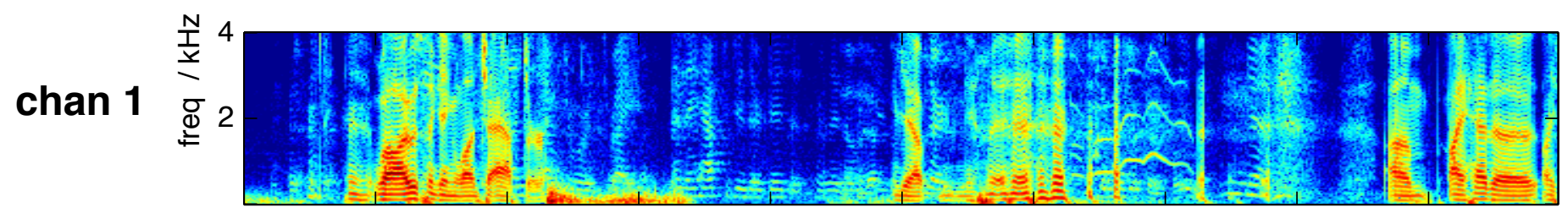

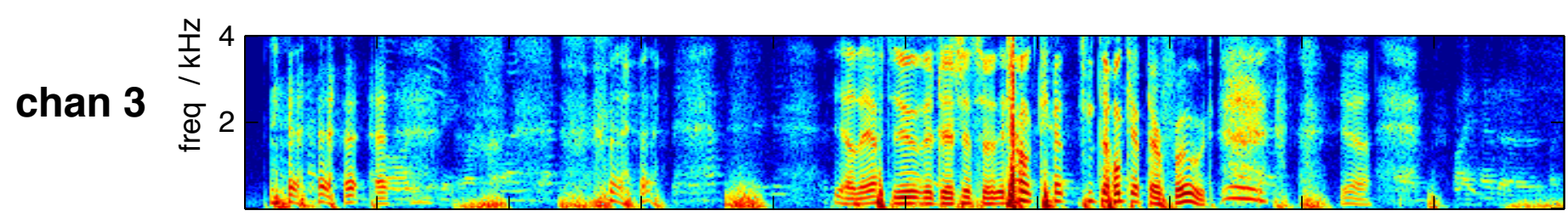
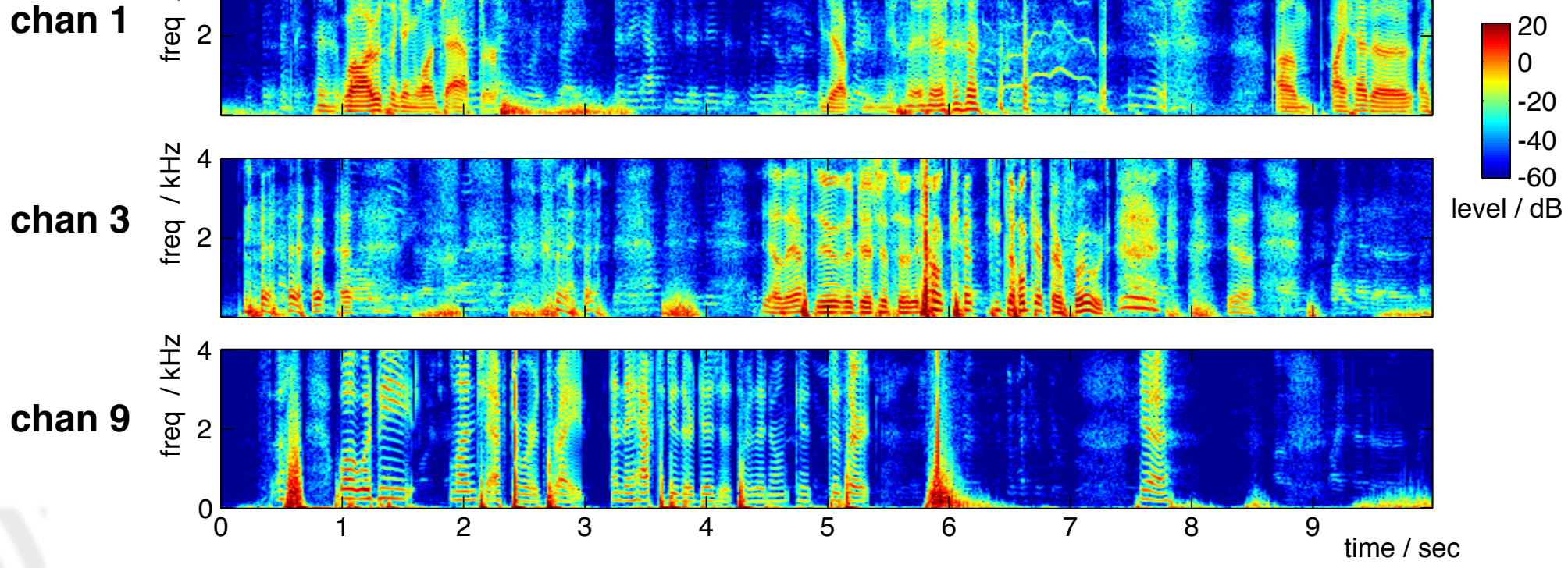

Speech Separation - Dan Ellis $2005-1 /-28-2 / 35$

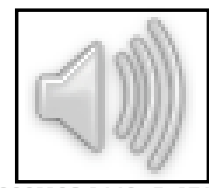




\section{Speech Separation Scenarios}

- Interactive voice systems

o human-level understanding is expected

- Speech prostheses

o crowds: \# I complaint of hearing aid users

- Archive analysis

o identifying and isolating speech
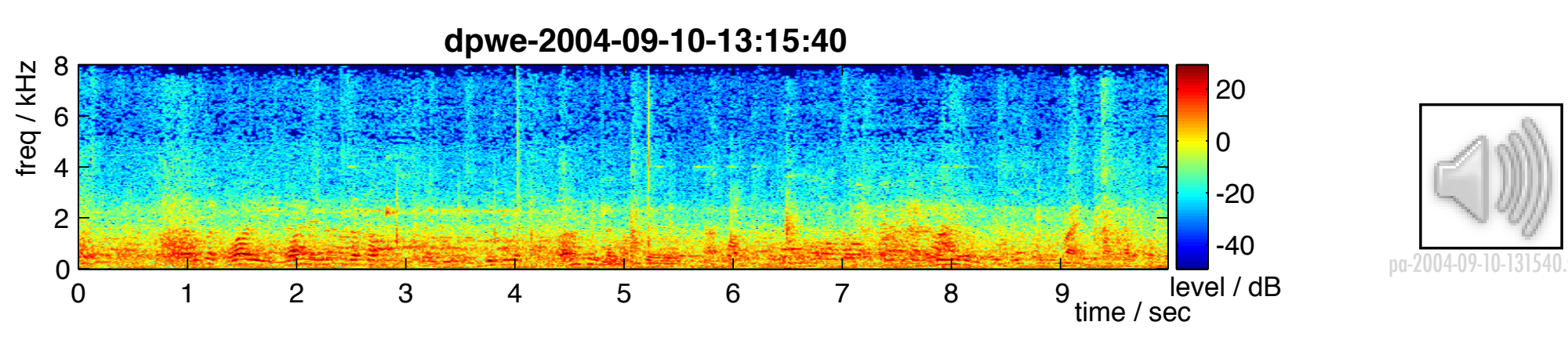

- Surveillance... 


\section{How Can We Separate?}

- By between-sensor differences (spatial cues) - 'steer a null' onto a compact interfering source

- By finding a 'separable representation'

o spectral? but speech is broadband

o periodicity? maybe - for voiced speech

o something more signal-specific...

- By inference (based on knowledge/models) o speech is redundant

$\rightarrow$ use part to guess the remainder 


\section{Outline}

\section{The Speech Separation problem}

2. Human Performance

o scene analysis

o speech separation by location

- speech separation by voice characteristics

3. Source Separation

4. Source Inference

5. Concluding Remarks 


\section{Auditory Scene Analysis}

- Listeners organize sound mixtures into discrete perceived sources based on within-signal cues (audio $+\ldots$...)

o common onset

+ continuity

o harmonicity

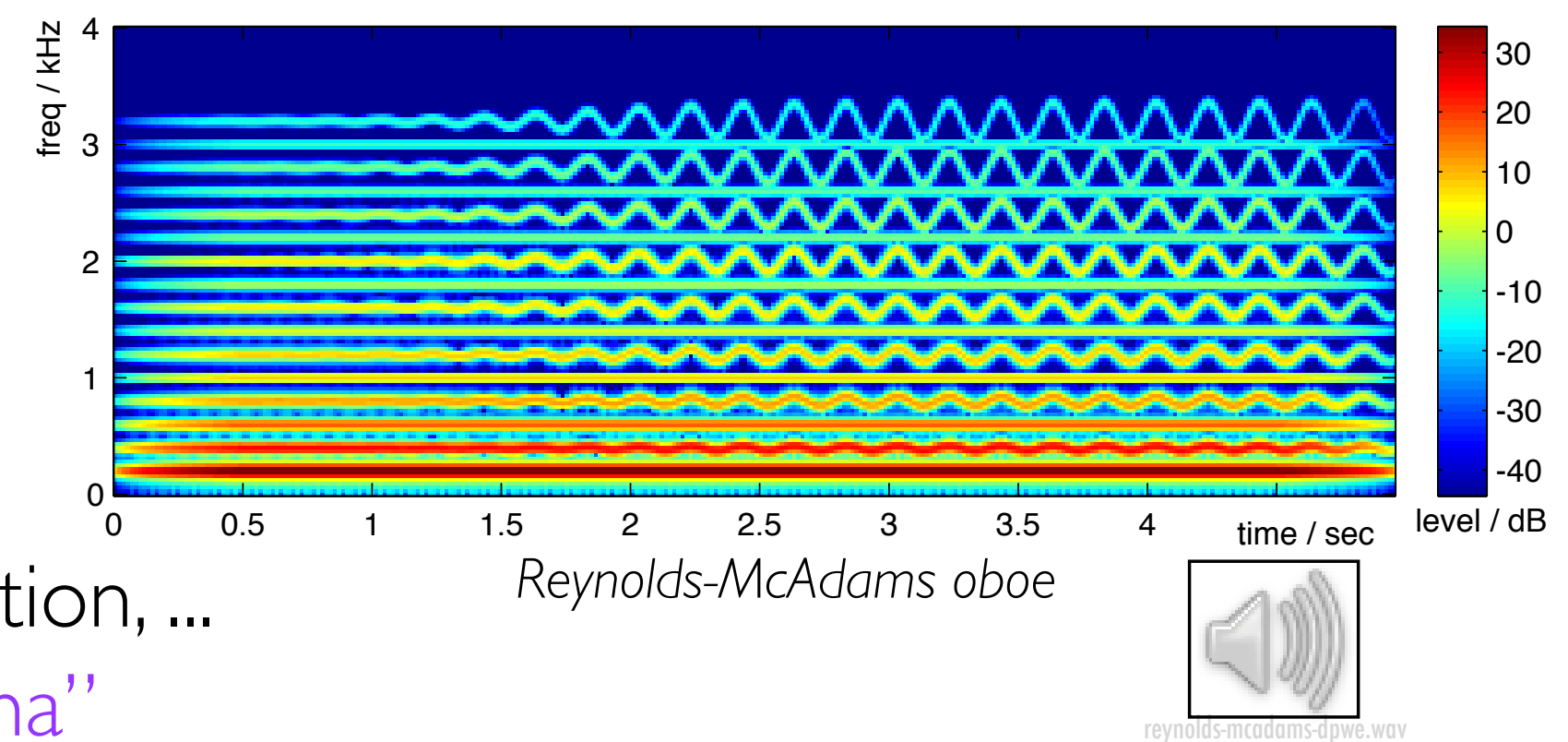

o spatial, modulation, ...

o learned "schema"

Dan
...) 


\section{Speech Mixtures: Spatial Separation}

Brungart et al.'02

- Task: Coordinate Response Measure - "Ready Baron go to green eight now"

- 256 variants, 16 speakers

o correct = color and number for "Baron"

- Accuracy as a function of spatial separation:

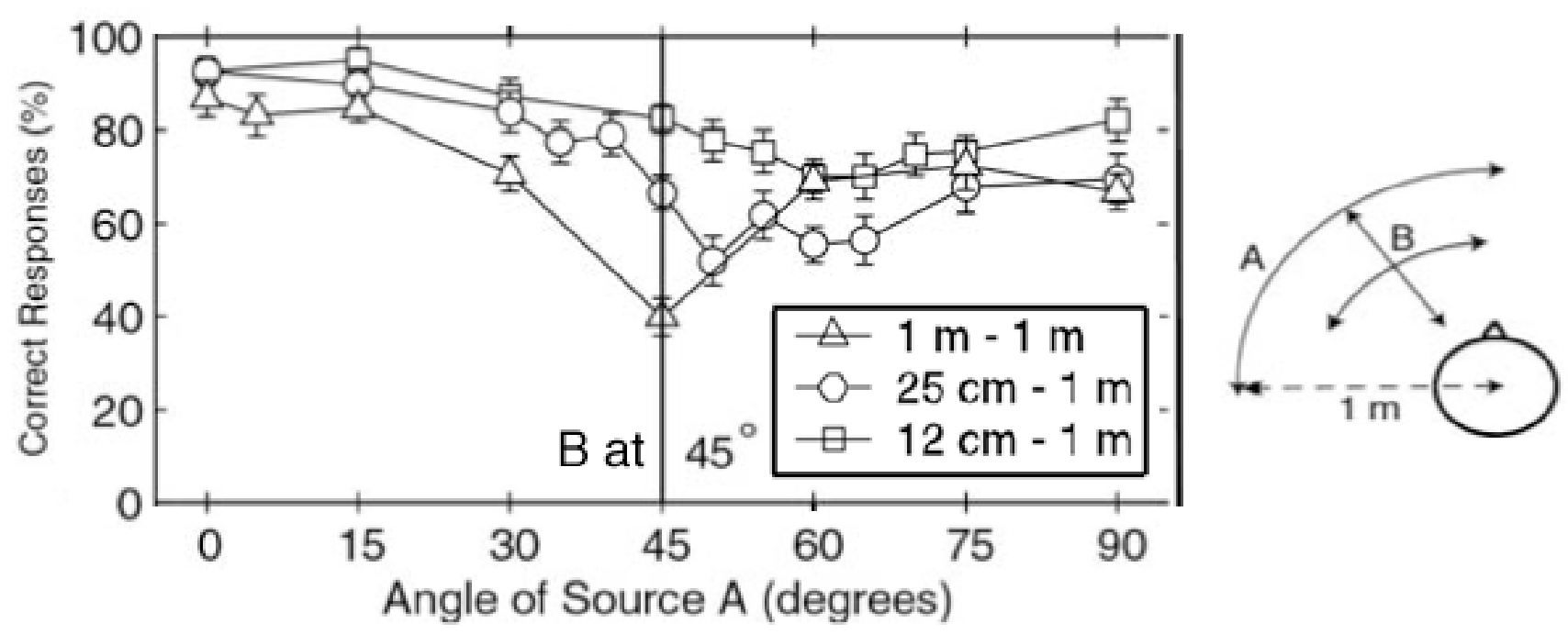

Lab

o A, B same speaker 


\section{Separation by Vocal Differences}

- CRM varying the level and voice character o (same spatial location)

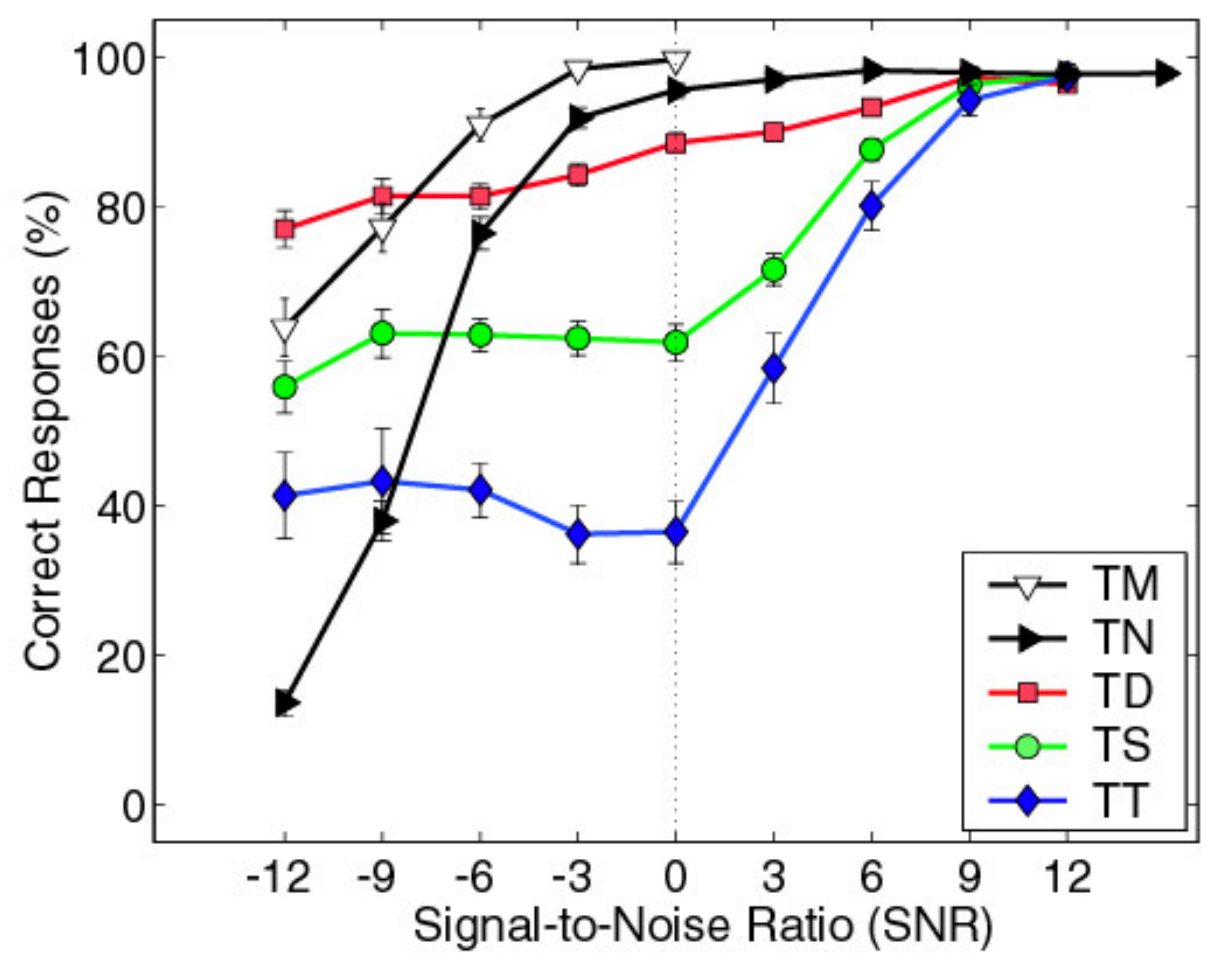

o energetic vs. informational masking 


\section{Varying the Number of Voices}

- Two voices OK;

More than two voices harder

o (same spatial origin)

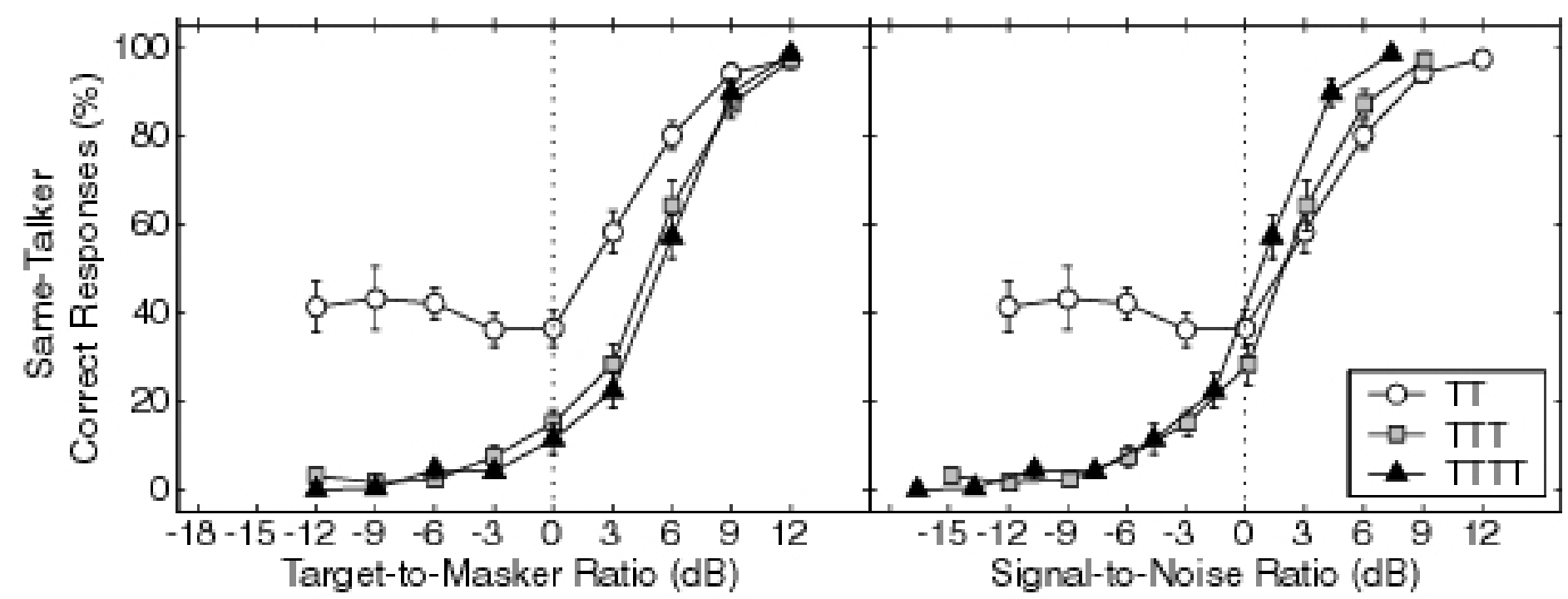

o mix of $N$ voices tends to speech-shaped noise... 


\section{Outline}

\section{The Speech Separation problem}

2. Human Performance

3. Source Separation

o Independent Component Analysis

- Computational Auditory Scene Analysis

4. Source Inference

5. Concluding Remarks 


\section{Machine Separation}

- Problem: Features of combinations are not combinations of features

o voice is easy to characterize when in isolation

o redundancy needed for real-world communication
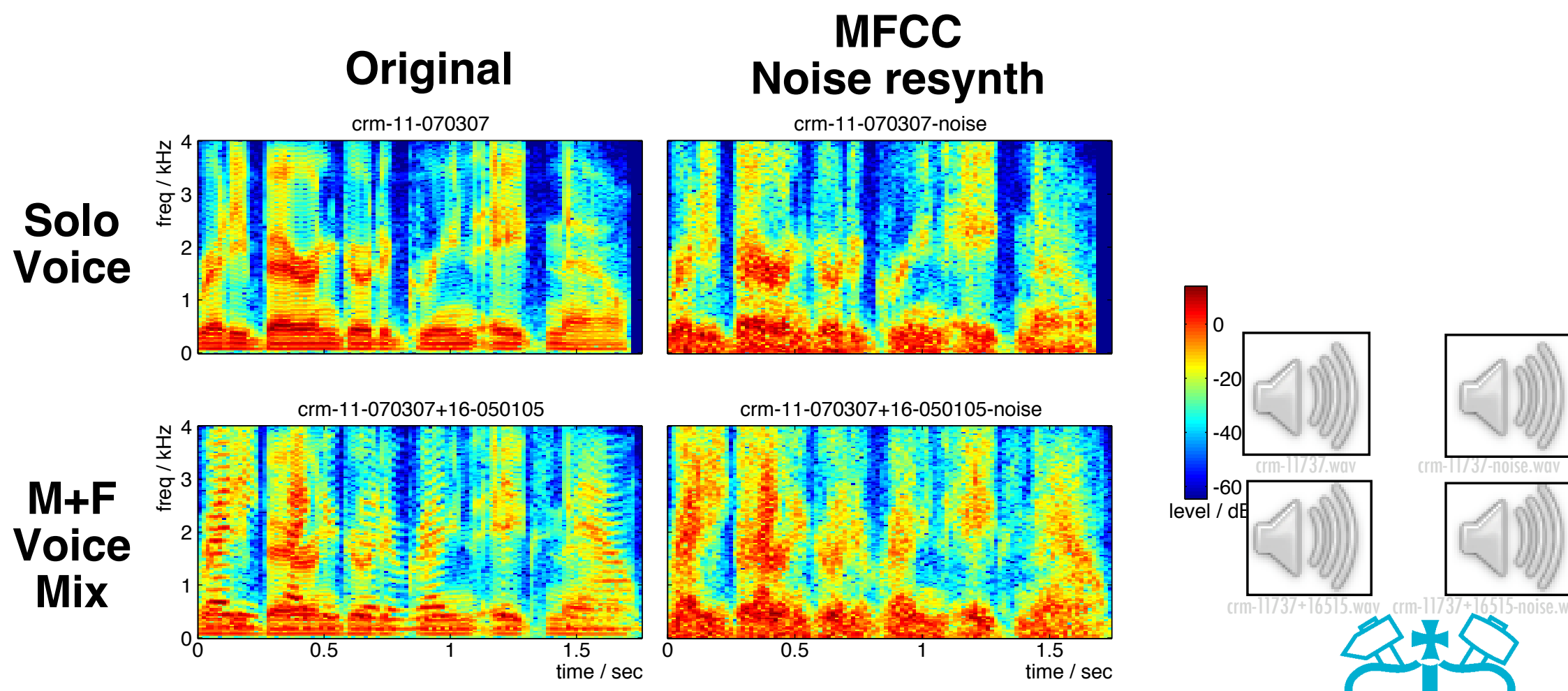

Speech Separation - Dan Ellis

$2005-||-28-|| / / 35$ 


\section{Separation Approaches}

\section{ICA}

- Multi-channel

- Fixed filtering

- Perfect separation

- maybe!

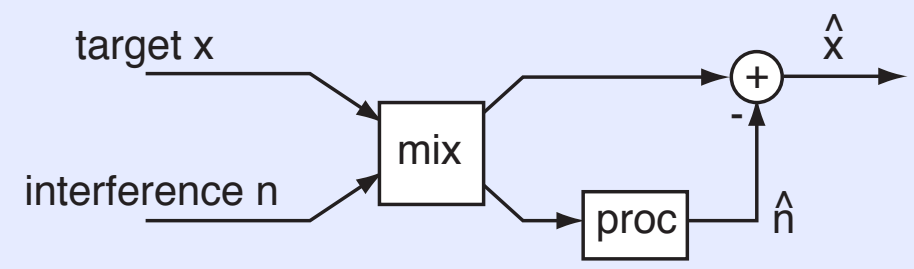

CASA / Model-based

- Single-channel

- Time-varying filtering

- Approximate

Separation

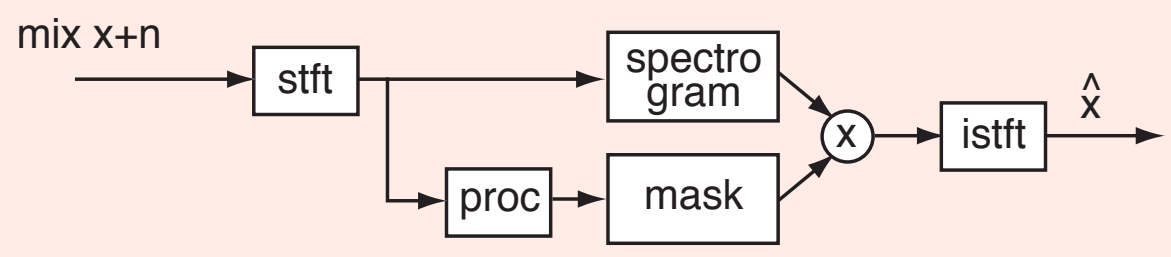

- Very different approaches!

Lab 


\section{Independent Component Analysis}

- Central idea:

Search unmixing space to maximize independence of outputs

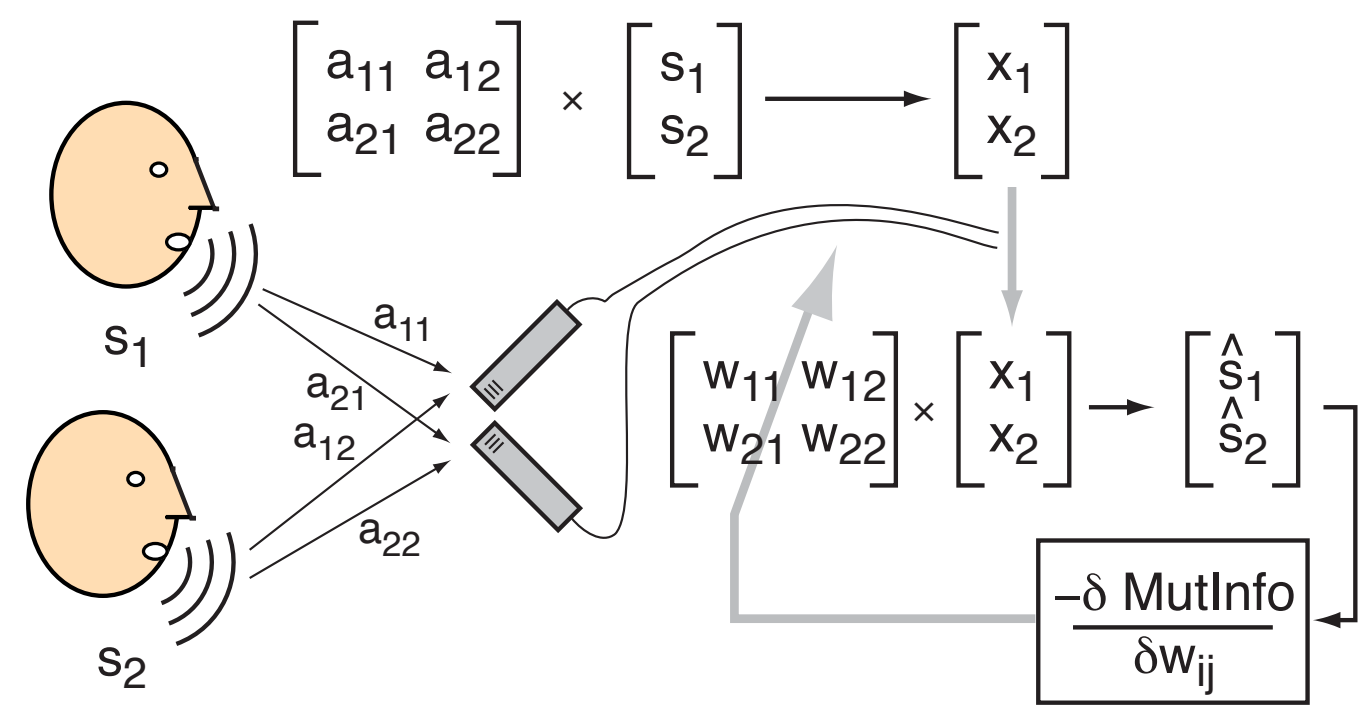

o simple mixing

$\rightarrow$ a good solution (usually) exists 


\section{ICA Limitations}

- Cancellation is very finicky o hard to get more than $\sim 10 \mathrm{~dB}$ rejection
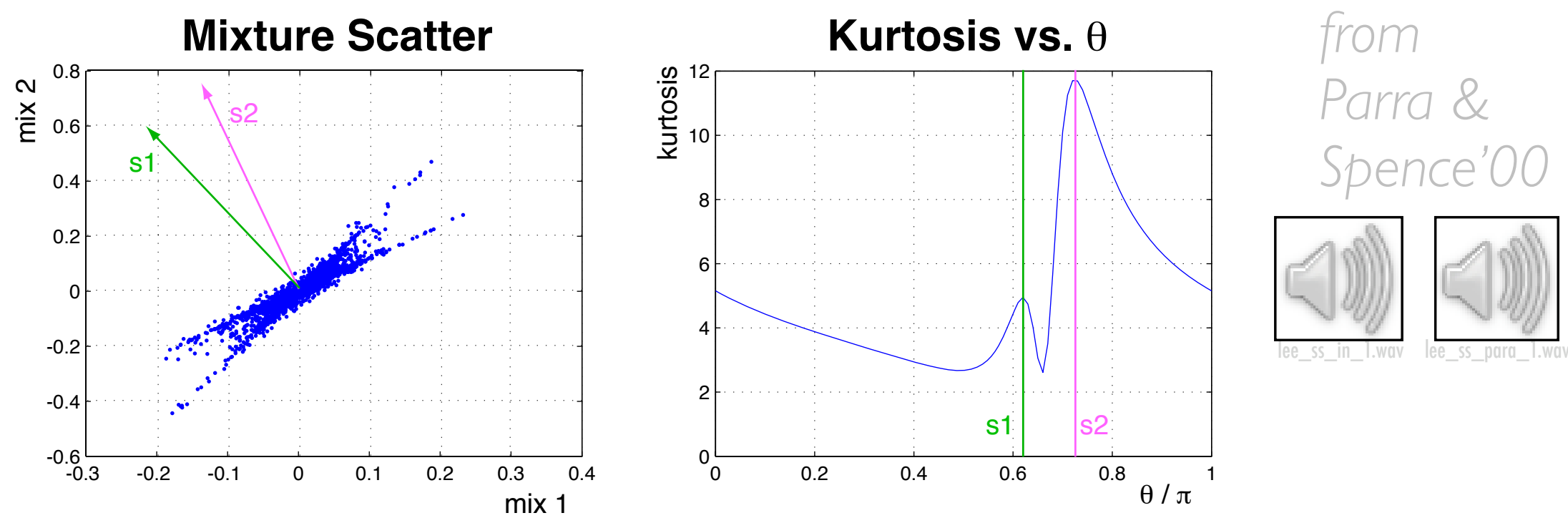

- The world is not instantaneous, fixed, linear o subband models for reverberation o continuous adaptation

- Needs spatially-compact interfering sources 


\section{Computational Auditory Scene Analysis}

- Central idea:

Segment time-frequency into sources based on perceptual grouping cues

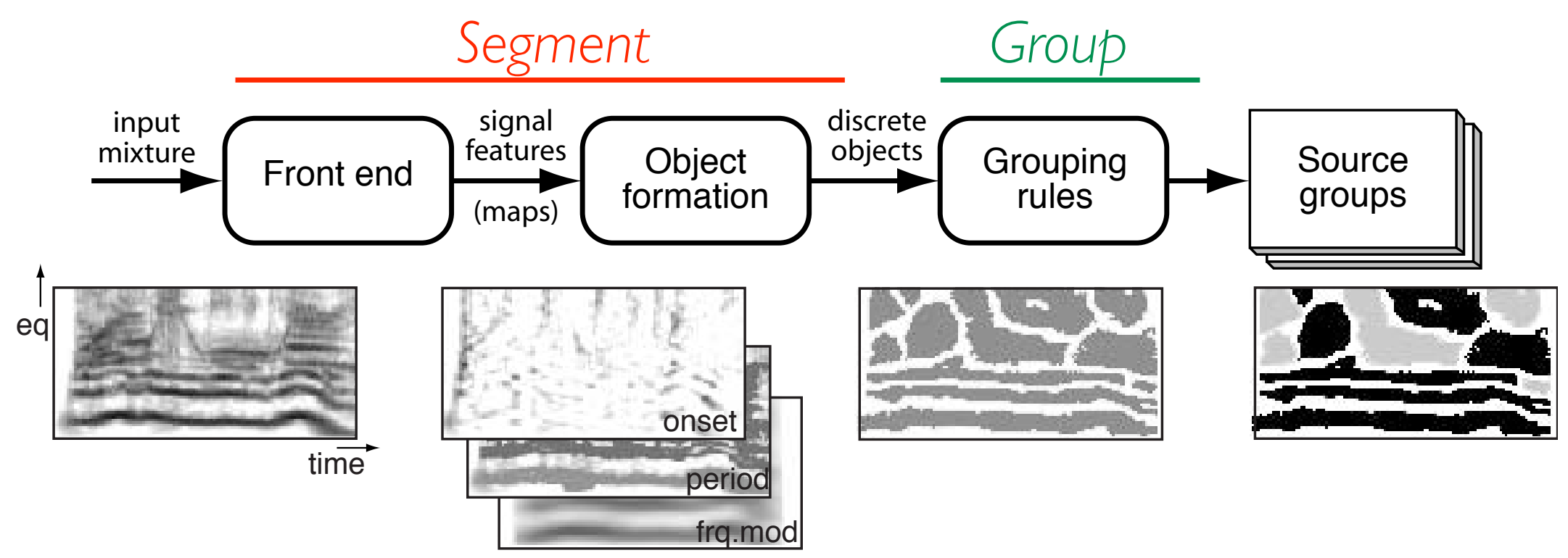

○ ... principal cue is harmonicity 


\section{CASA Preprocessing}

- Correlogram: a 3rd "periodicity" axis

o envelope of wideband channels follows pitch

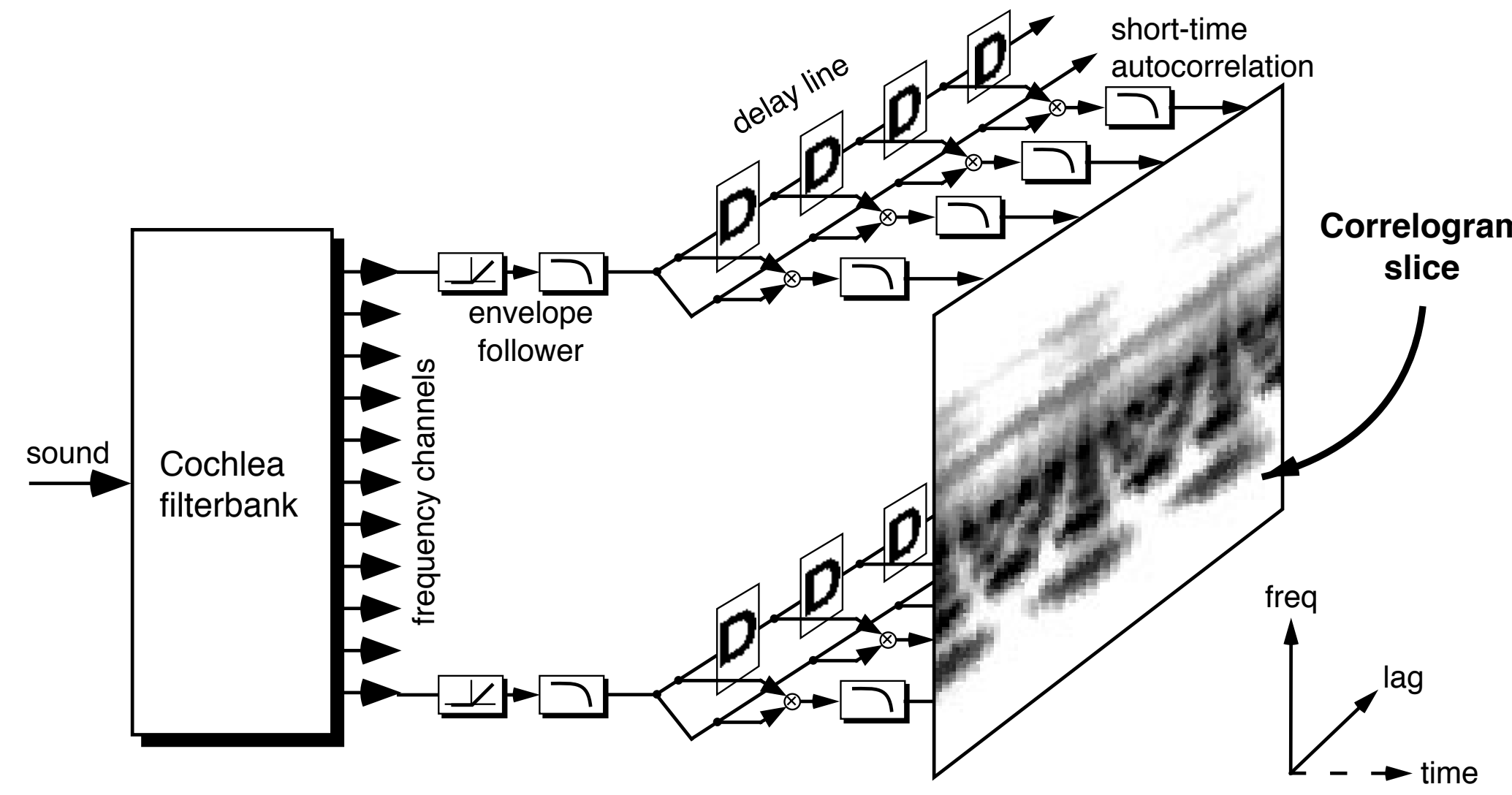

$\mathrm{Lab}$

o c/w Modulation Filtering [Schimmel \& Atlas '05] 


\section{Time-Frequency (T-F) Masking}

- "Local Dominance" assumption
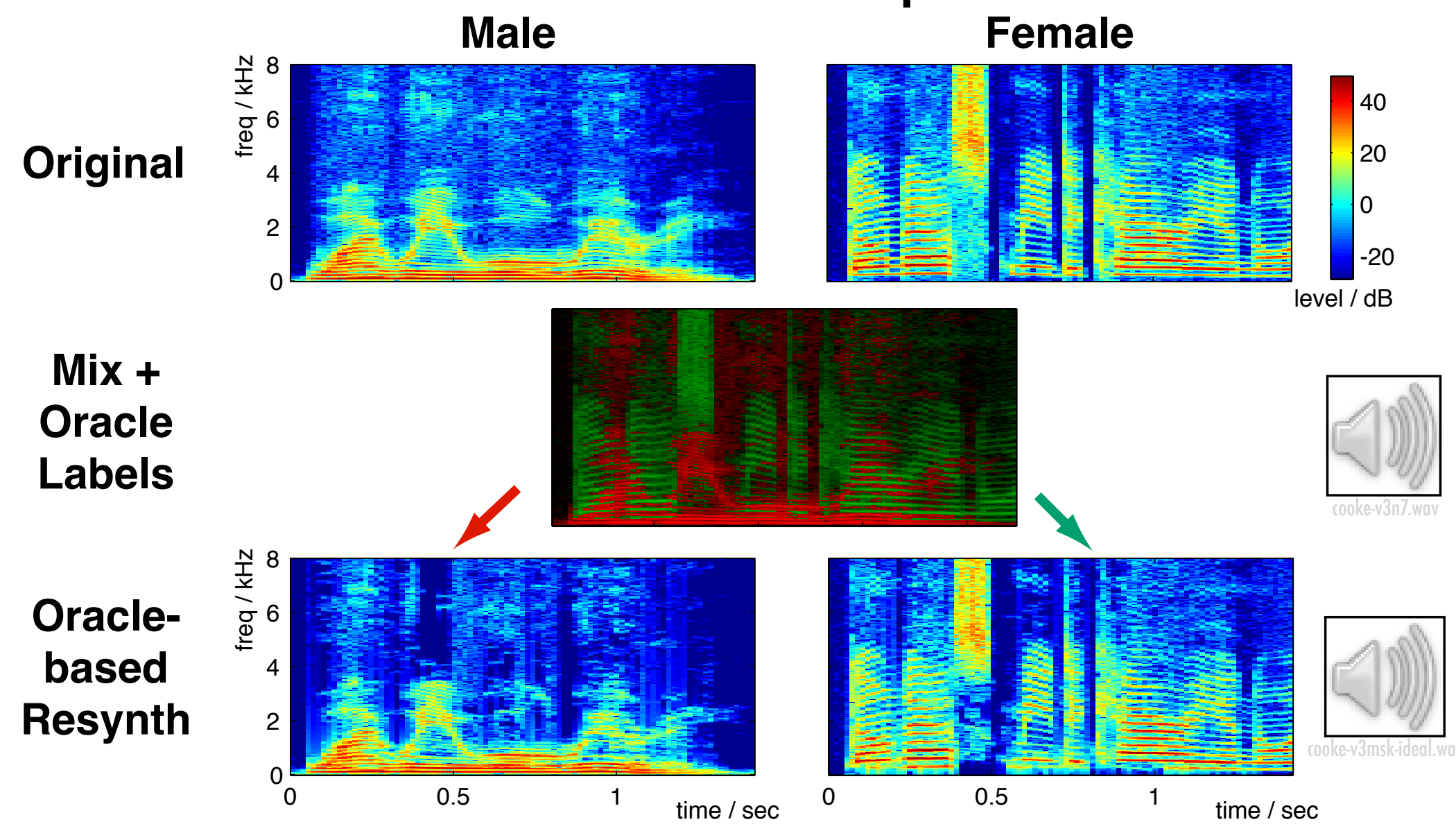

- oracle masks are remarkably effective!
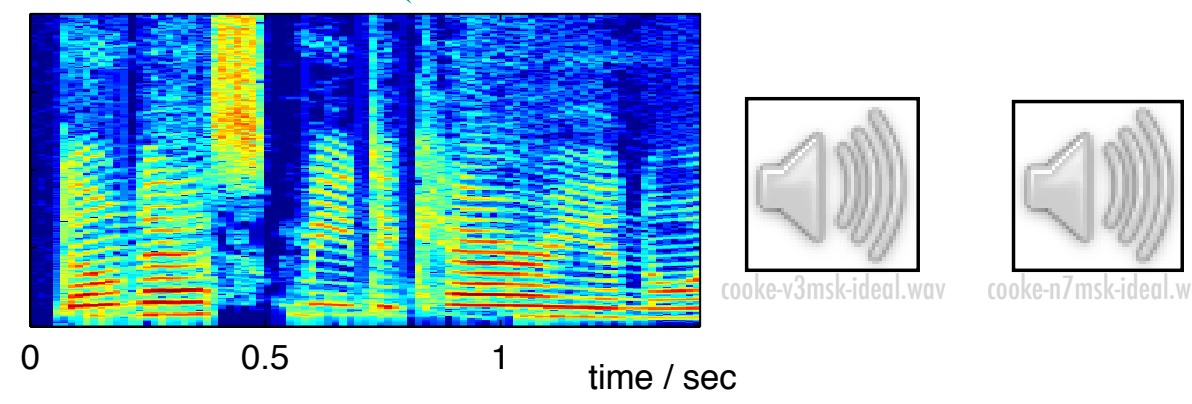

Lab

$\frac{\mathrm{O} \mid \text { mix }-\max (\text { male }, \text { }}{\text { Speech Separation - Dan Ellis }}$

$<3 \mathrm{~dB}$ for $\sim 80 \%$ of cells 1 


\section{CASA limitations}

- Driven by local features

o problems with aperiodic sources...

- Limitations of T-F masking

o need to identify single-source regions

o cannot undo overlaps - leaves gaps
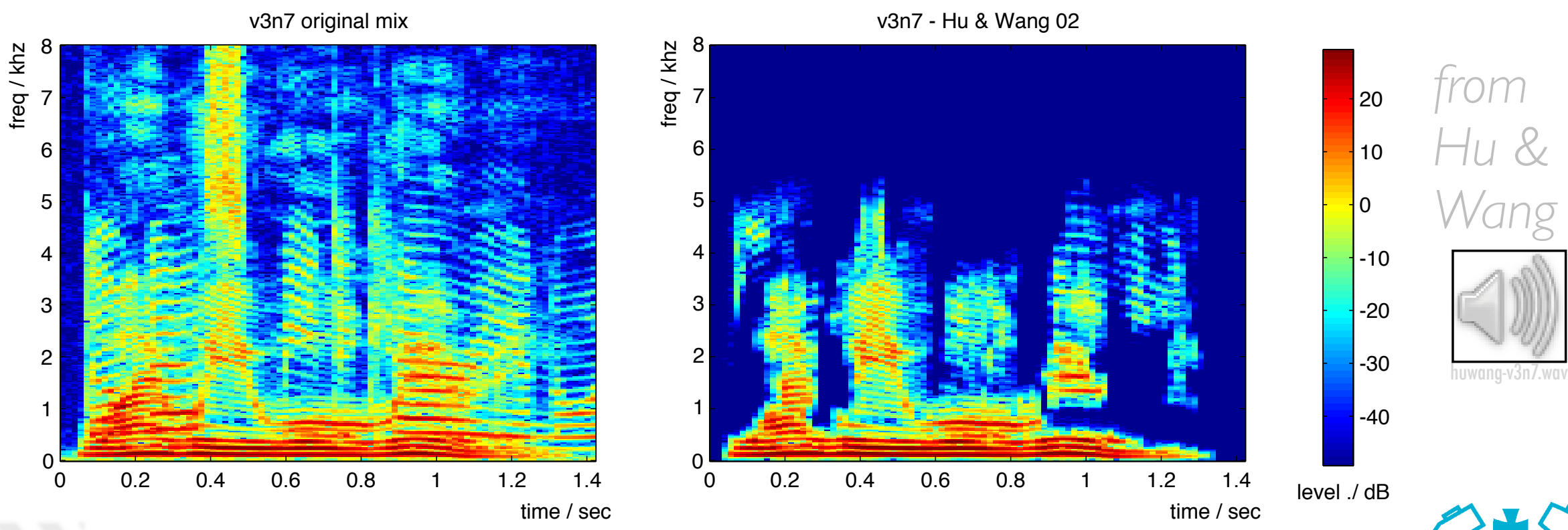

Lab

Speech Separation - Dan Ellis

$2005-||-28-18 / 35$ 


\section{Combining Spatial + T-F Masking}

- T-F masks based on inter-channel properties [Yilmaz \& Rickard' '04] (O2)J GOJ)

o multiple channels make CASA-like masks better

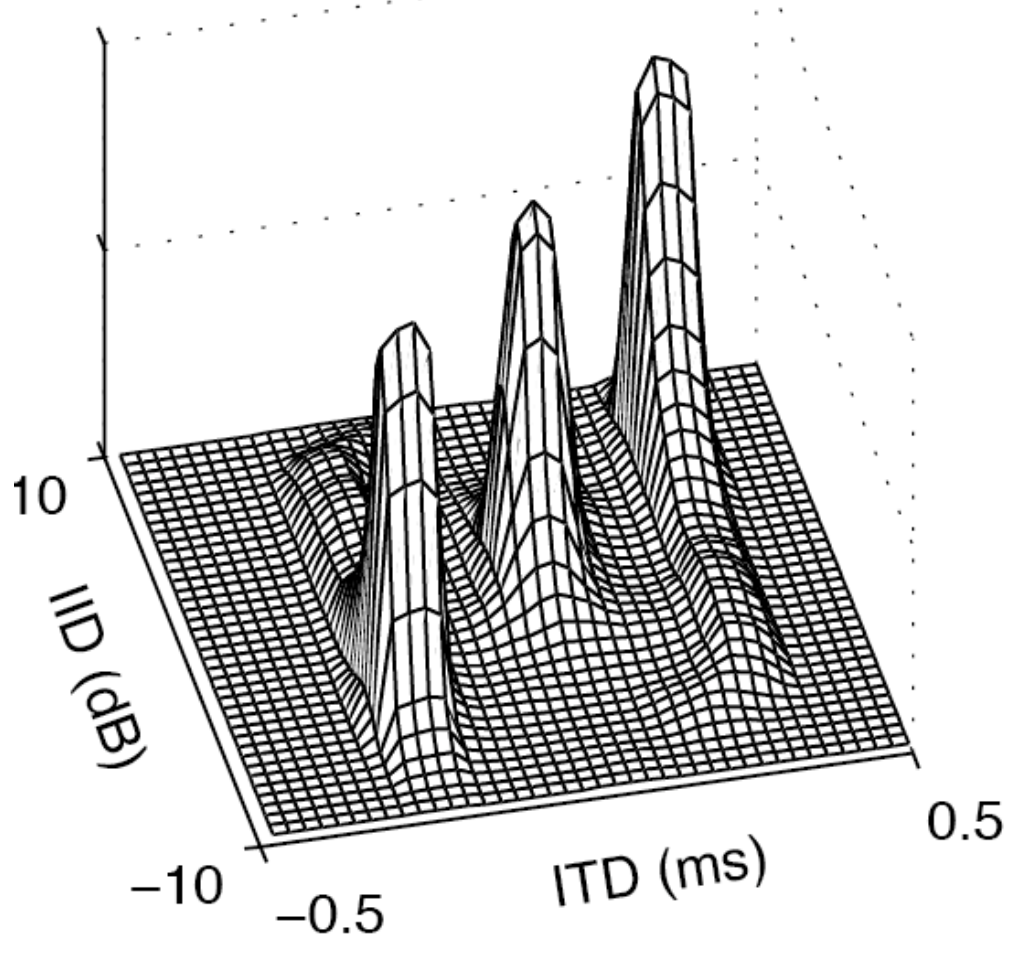

- T-F masking after ICA

- cancellation can remove energy within T-F cells 


\section{Outline}

\section{The Speech Separation problem}

2. Human Performance

3. Source Separation

4. Source Inference

- Separation vs. inference

- Model-based separation

- Speech Fragment Decoding

5. Concluding Remarks 


\section{Separation vs. Inference}

- Ideal separation is rarely possible

o i.e. no projection can completely remove overlaps

- Overlaps $\Rightarrow$ Ambiguity

o scene analysis = find "most reasonable" explanation

- Ambiguity can be expressed probabilistically

o i.e. posteriors of sources $\left\{S_{i}\right\}$ given observations $X$ :

$$
P\left(\left\{S_{i}\right\} \mid X\right) \propto P\left(X \mid\left\{S_{i}\right\}\right) P\left(\left\{S_{i}\right\}\right)
$$

combination physics source models

- Better source models $\rightarrow$ better inference o.. learn from examples? 


\section{Model-Based Separation}

- Central idea:

Employ strong learned constraints

to disambiguate possible sources

$\circ\left\{S_{i}\right\}=\operatorname{argmax}_{S i} P\left(X \mid\left\{S_{i}\right\}\right)$

- e.g. fit speech-trained Vector-Quantizer to mixed spectrum:
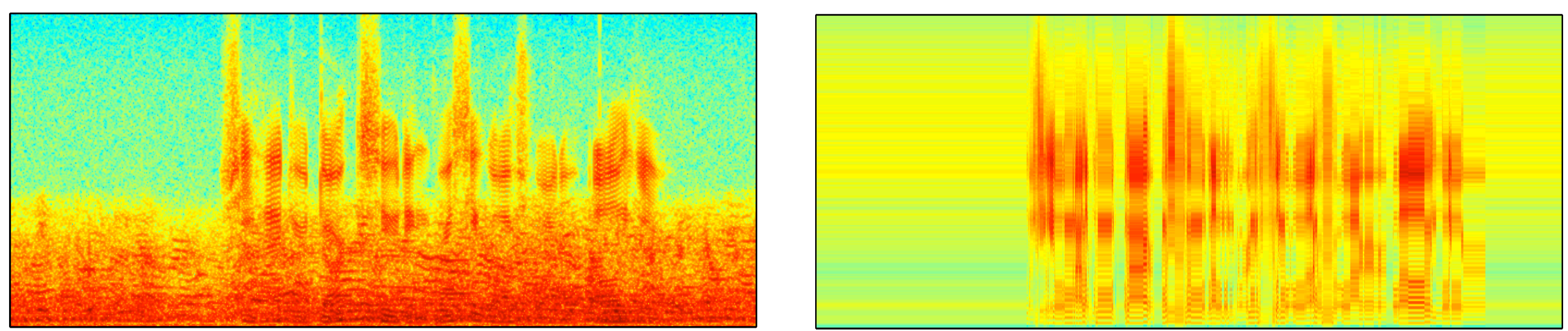

o separate via T-F mask (again) 


\section{Can Models Do CASA?}

- Source models can learn harmonicity, onset - ... to subsume rules/representations of CASA

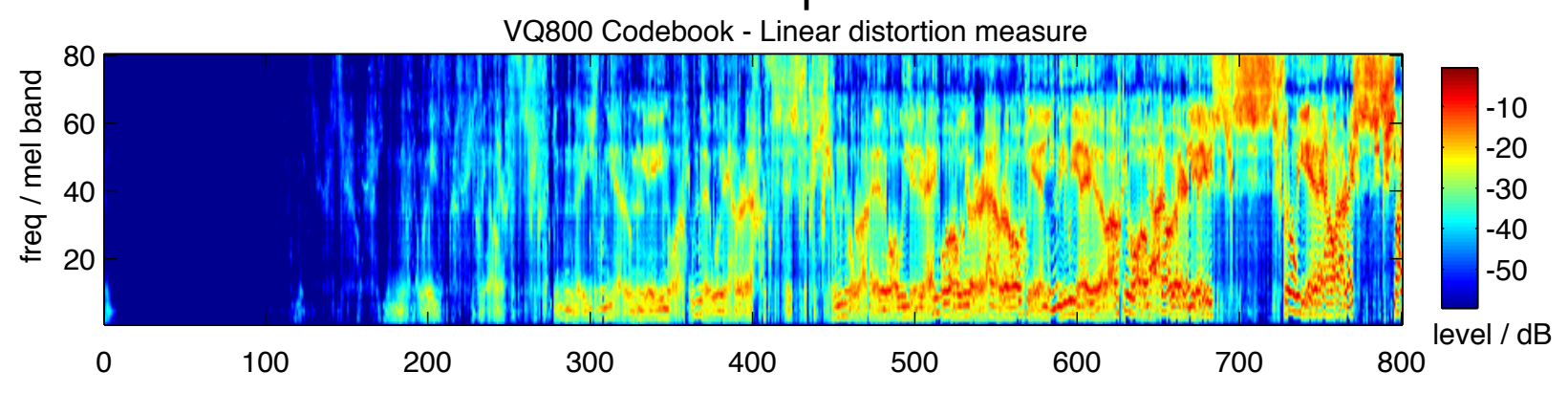

o can capture spatial info too [Pearlmutter \& Zador'04]

- Can also capture sequential structure

o e.g. consonants follow vowels

○... like people do?

- But: need source-specific models ... for every possible source

Lab o use model adaptation? [Ozerov et al. 2005] 


\section{Separation with ASR Models}

- Drive separation engine to match outputs to existing speech models

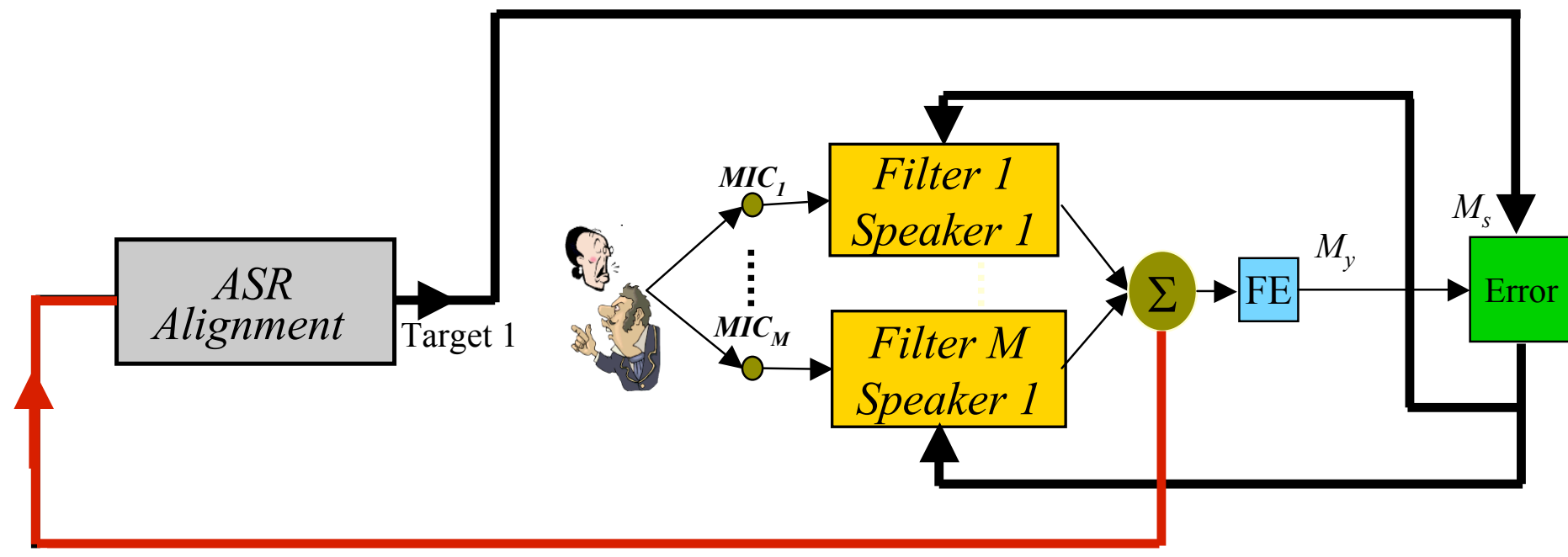

- ASR includes a very detailed source model 


\section{Separation or Description?}

- Are isolated waveforms required?

- clearly sufficient, but may not be necessary

o not part of perceptual source separation!

- Integrate separation with application?

o e.g. speech recognition
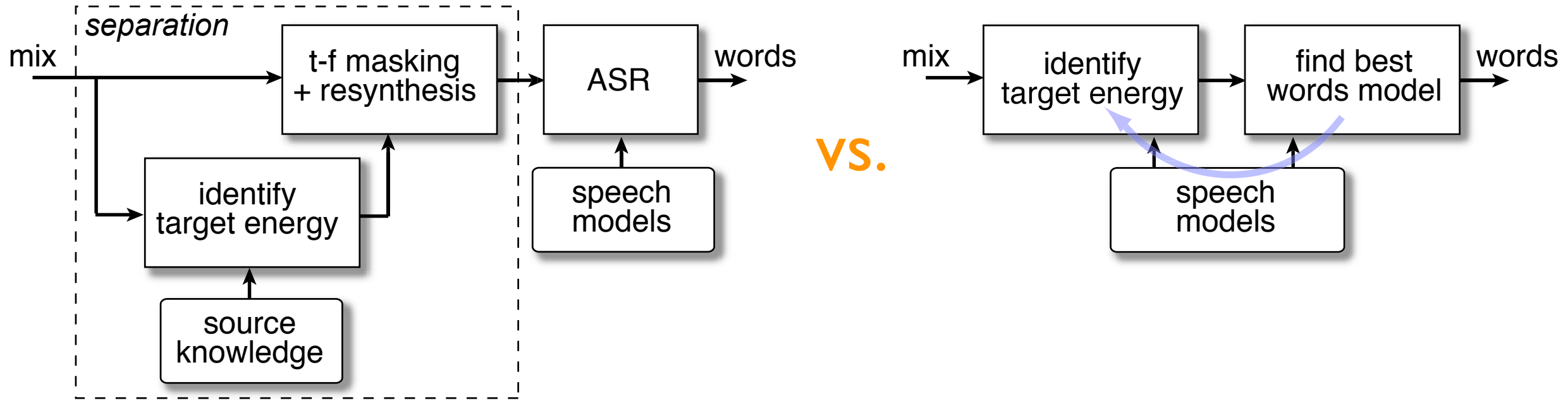

Lab

owords output = abstract description of signal 


\section{Missing Data Recognition}

- Speech models $p(x \mid M)$ are multidimensional...

o need values for all dimensions to evaluate $p(\bullet)$

- But: can make inferences given just a subset of dimensions $x_{k}$ ○ $\quad p\left(x_{k} \mid M\right)=\int p\left(x_{k}, x_{u} \mid M\right) d x_{u}$

- Hence, missing data recognition:
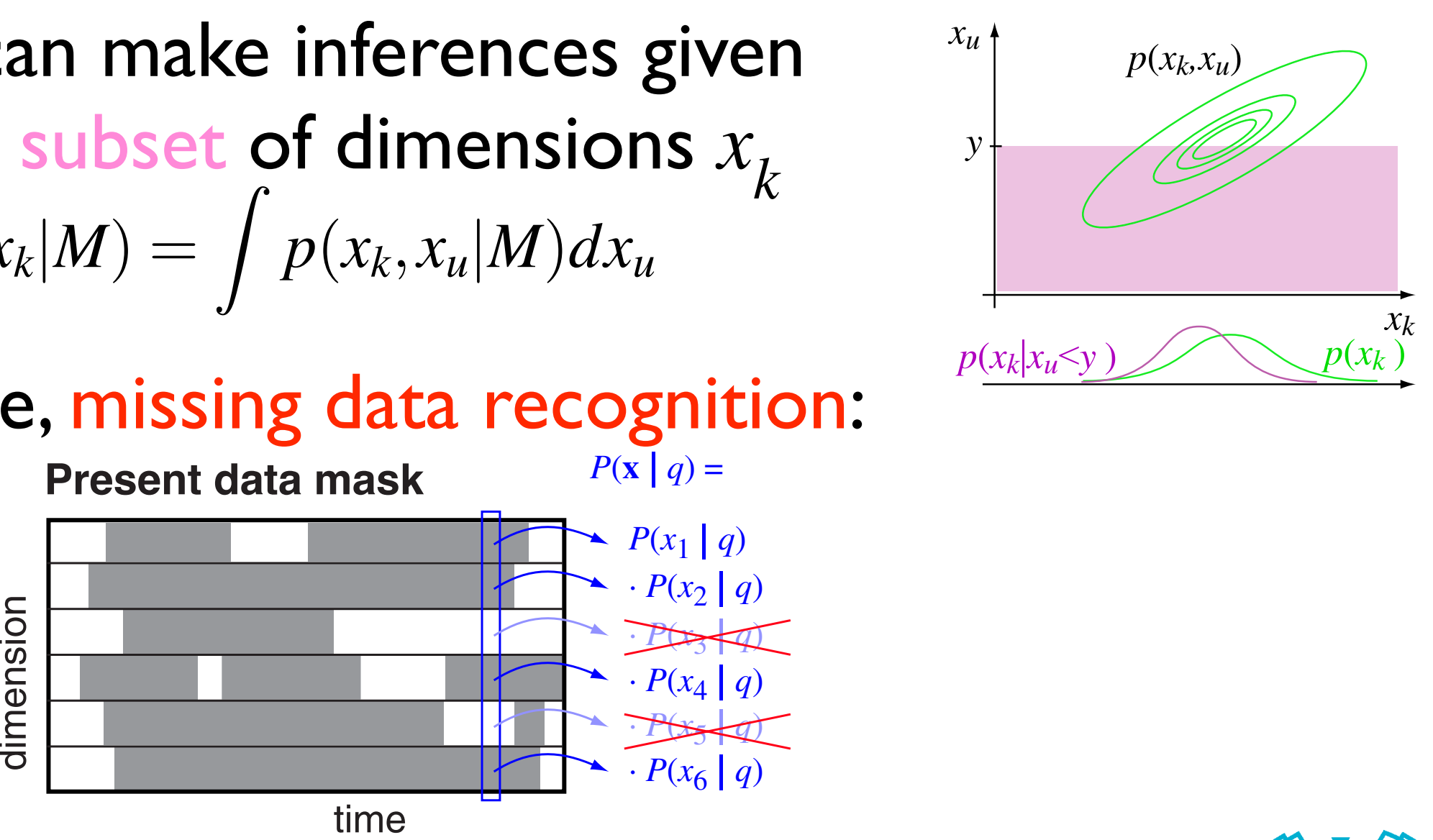

Lab o hard part is finding the mask (segregation) 


\section{The Speech Fragment Decoder}

- Match 'uncorrupt' spectrum to ASR models using missing data

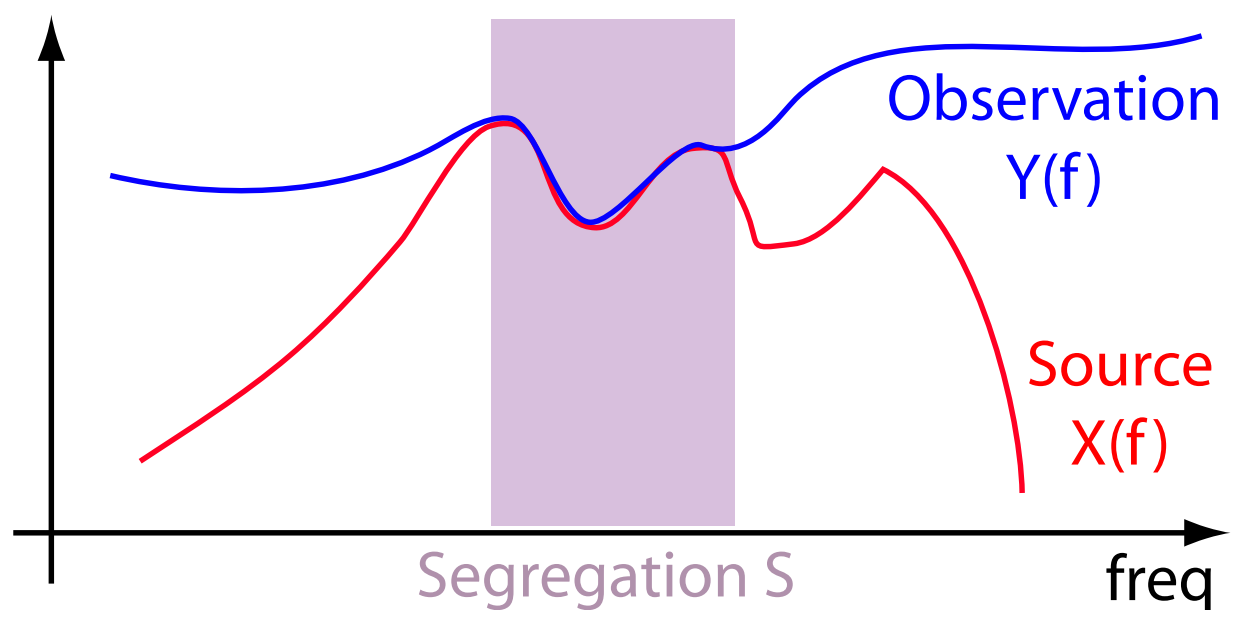

- Joint search for model $M$ and segregation $S$ to maximize:

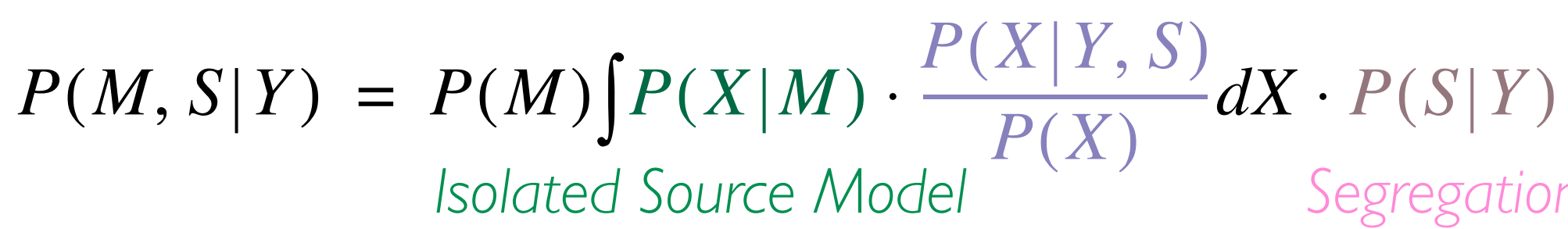

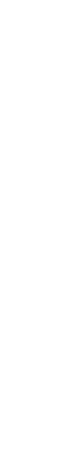




\section{Using CASA cues}

$P(M, S \mid Y)=P(M) \int P(X \mid M) \cdot \frac{P(X \mid Y, S)}{P(X)} d X \cdot P(S \mid Y)$

- CASA can help search

o consider only segregations made from CASA chunks

- CASA can rate segregation

o construct $P(S \mid Y)$ to reward CASA qualities:

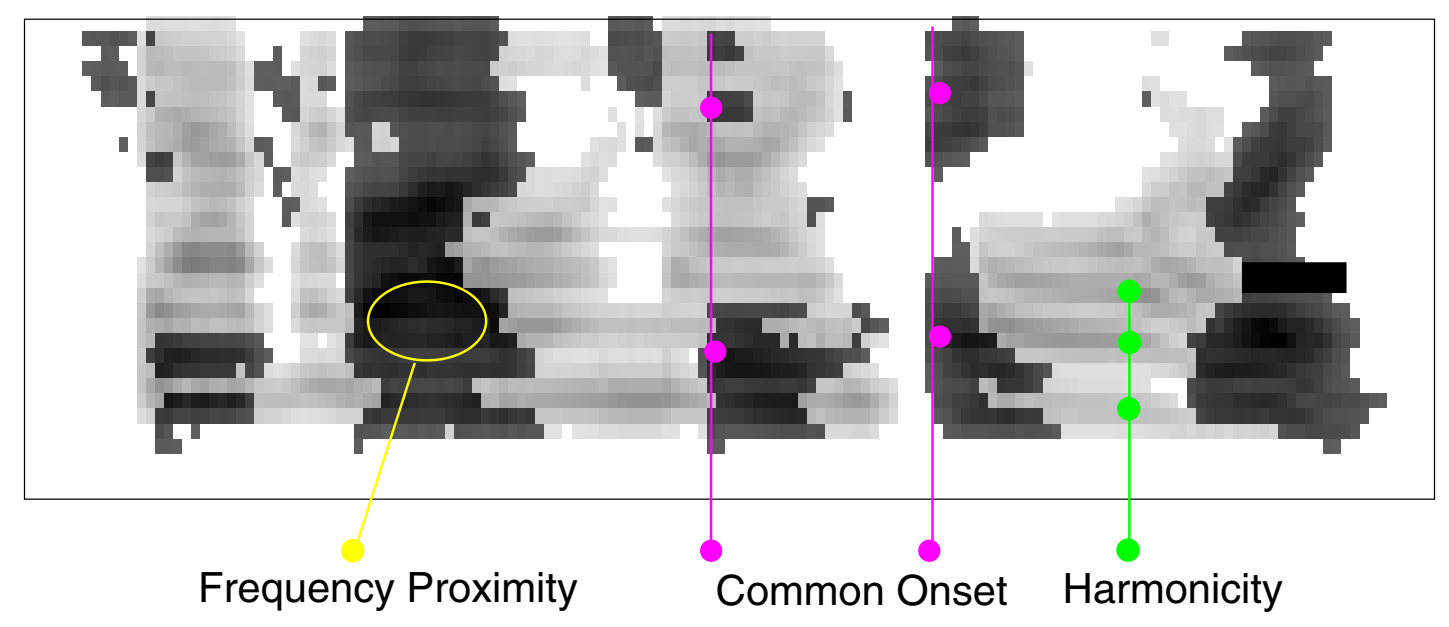

Speech Separation - Dan Ellis $2005-||-28-28 / 35$ 


\section{Speech-Fragment Recognition}

- CASA-based fragments give extra gain over missing-data recognition

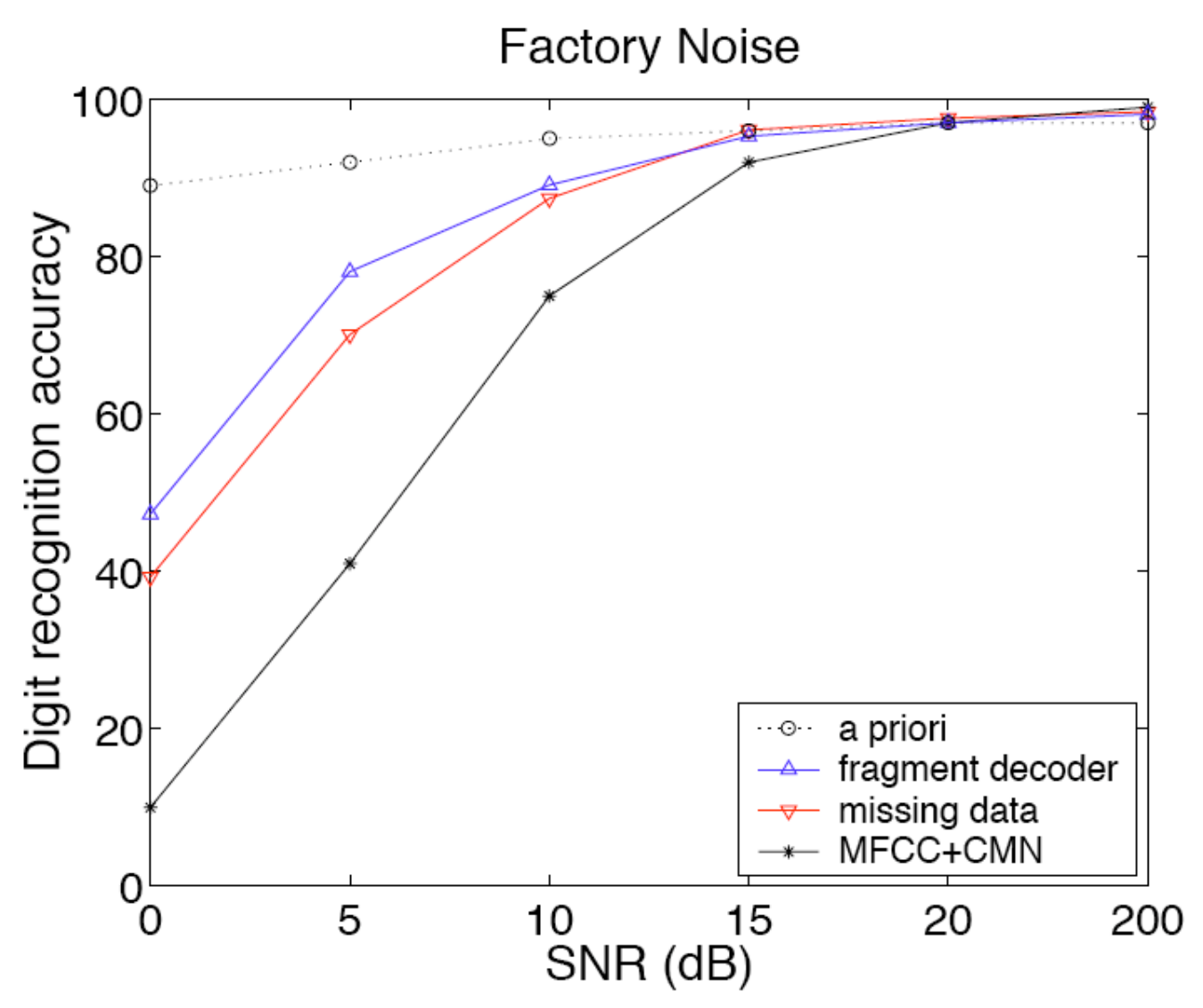




\section{Outline}

\section{The Speech Separation problem}

2. Human Performance

3. Source Separation

4. Source Inference

5. Concluding Remarks

o Evaluation

- Connecting to Perception 


\section{Evaluation}

- How to measure separation performance?

o depends what you are trying to do

- SNR?

o energy (and distortions) are not created equal o different nonlinear components

- Intelligibility?

o rare for nonlinear processing to improve intelligibility

o listening tests expensive

- ASR performance?

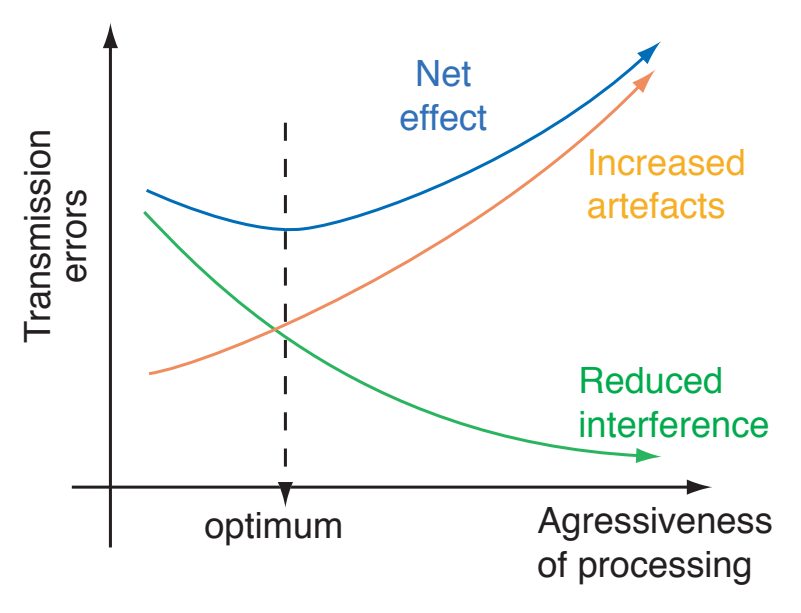

o separate-then-recognize too simplistic; ASR needs to accommodate separation 


\section{"Speech Separation Challenge"}

- Mixed and Noisy Speech ASR task defined by Martin Cooke and Te-Won Lee

o short, grammatically-constrained utterances:

<command:4><color:4><preposition:4><letter:25><number:10><adverb:4> e.g. "bin white at M 5 soon"

- Results to be presented at Interspeech'06 - http://www.dcs.shef.ac.uk/ martin/SpeechSeparationChallenge.htm

- See also "Statistical And Perceptual Audition" workshop

○ http://www.sapa2006.org/ 


\section{More Realistic Evaluation}

- Real-world speech tasks

o crowded environments

o applications: communication, command/control, transcription

- Metric o human intelligibility? o 'diarization' annotation (not transcription)
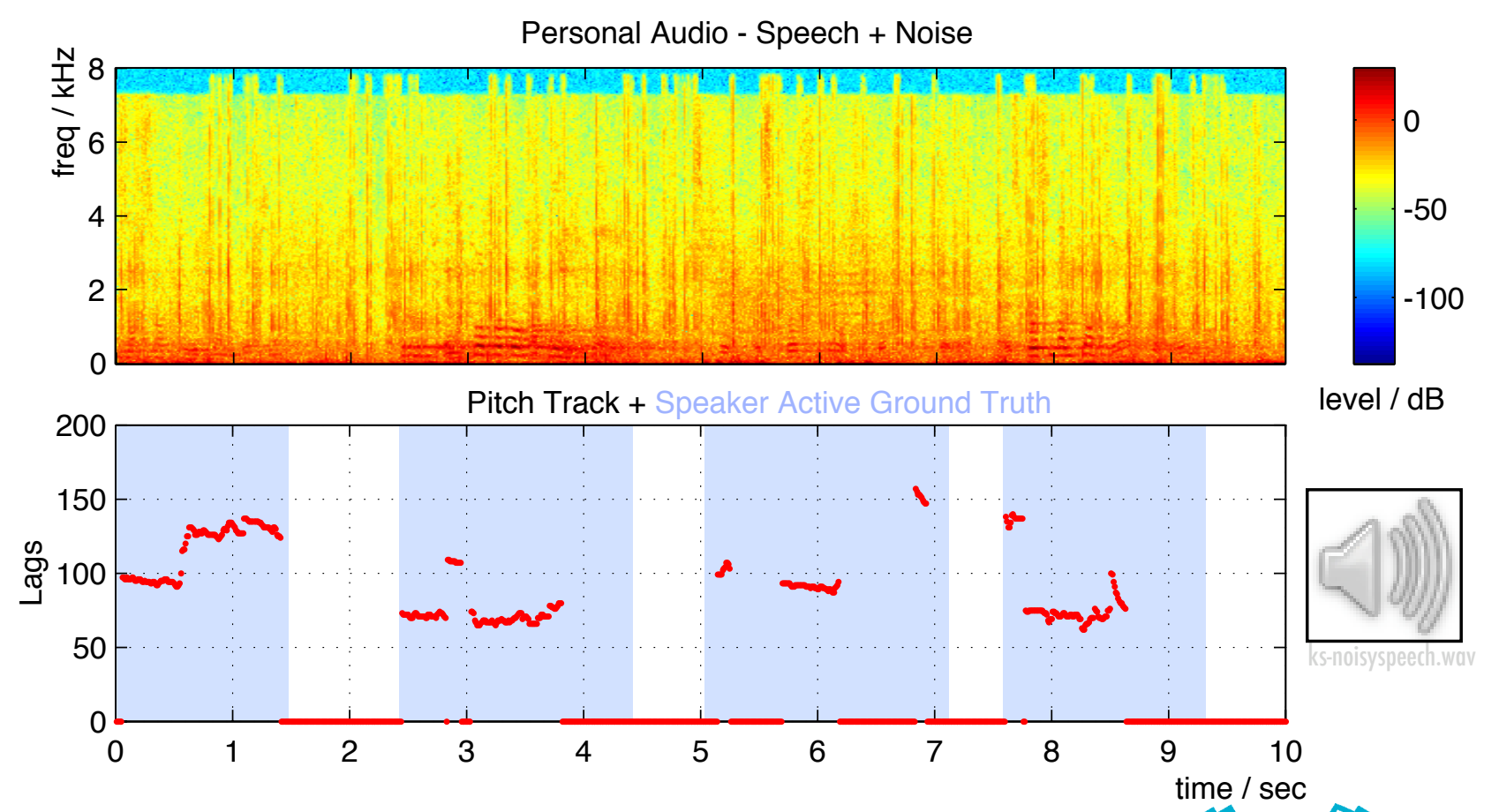

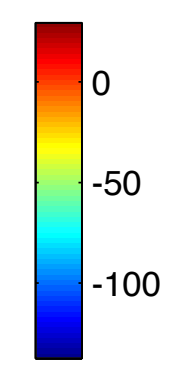

level / dB

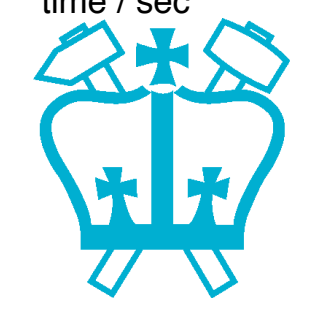




\section{Reconnecting to Perception}

- People are (still) much better at speech recognition, including mixtures

- Can we model human separation with ASR?

- "Glimpse model": MD ASR using oracle local SNR

- Listeners identify high SNR islands?

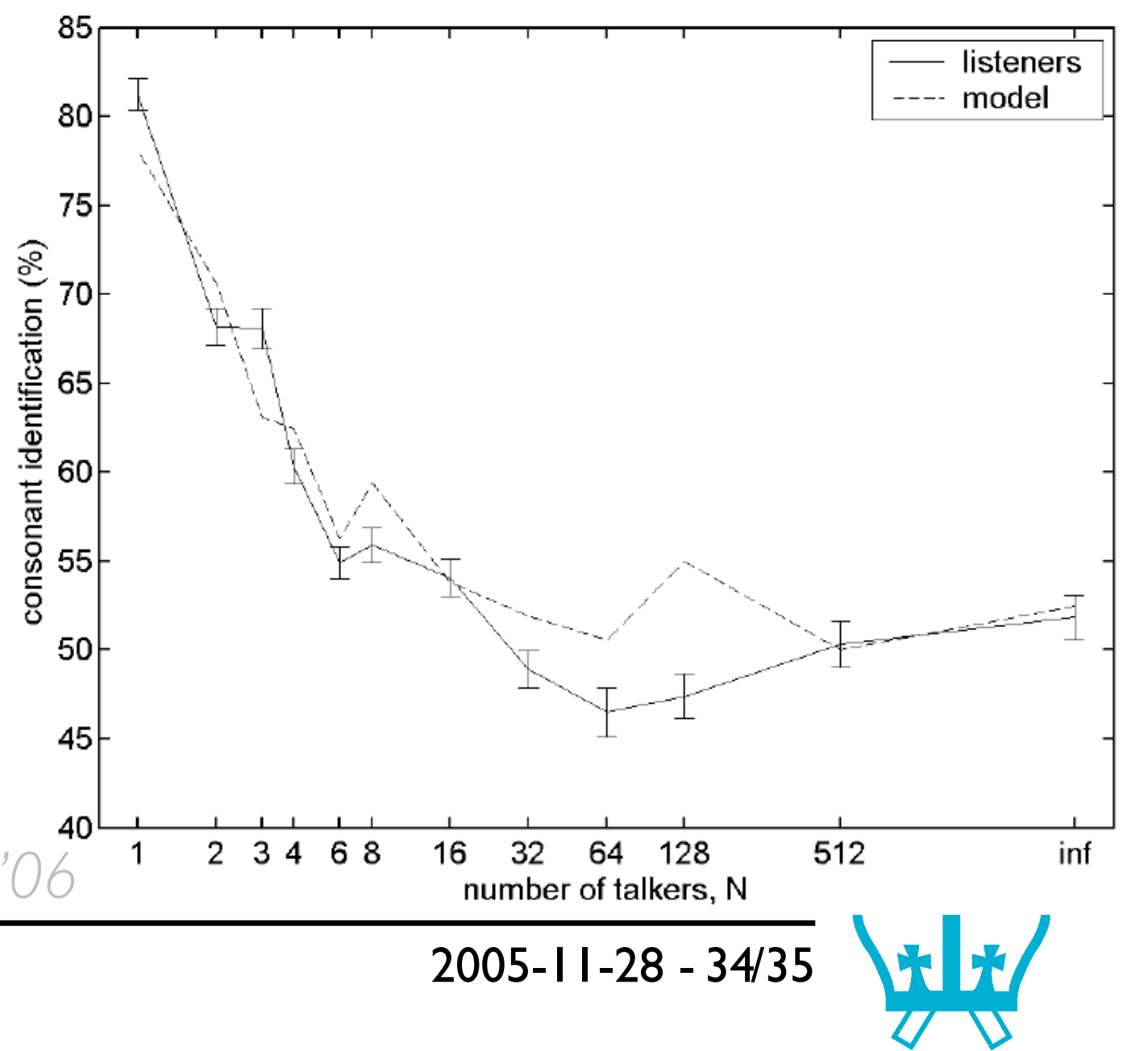




\section{Summary \& Conclusions}

- Listeners do well separating speech

o using spatial location

o using source-property variations

- Machines do less well

- difficult to apply enough constraints

o need to exploit signal detail

- Models capture constraints

o learn from the real world

o adapt to sources

- Inferring state ( $\approx$ recognition) is a promising approach to separation 


\section{Sources / See Also}

- NSF/AFOSR Montreal Workshops '03, '04

o www.ebire.org/speechseparation/

- labrosa.ee.columbia.edu/Montreal2004/

o as well as the resulting book...

- Hanse meeting:

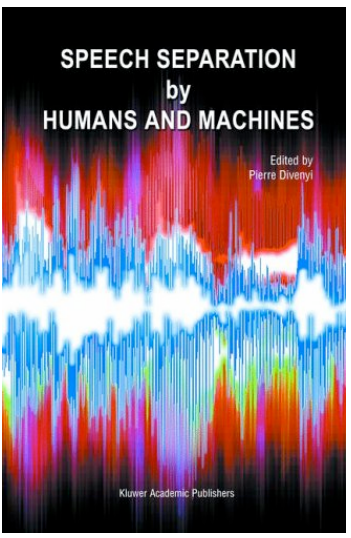

- www.lifesci.sussex.ac.uk/home/Chris Darwin/ Hanse/

- DeLiang Wang's ICASSP'04 tutorial

o www.cse.ohio-state.edu/ dwang/presentation.html

- Martin Cooke's NIPS'02 tutorial

o www.dcs.shef.ac.uk/ martin/nips.ppt 


\section{References I/2}

[Barker et al. '05] J. Barker, M. Cooke, D. Ellis, “Decoding speech in the presence of other sources,” Speech Comm. 45, 5-25, 2005.

[Bell \& Sejnowski '95] A. Bell \& T. Sejnowski, “An information maximization approach to blind separation and blind deconvolution,” Neural Computation, 7:I I29-I I59, 1995.

[Blin et al.'04] A. Blin, S.Araki, S. Makino, “A sparseness mixing matrix estimation (SMME) solving the underdetermined BSS for convolutive mixtures,” ICASSP, IV-85-88, 2004.

[Bregman '90] A. Bregman, Auditory Scene Analysis, MIT Press, 1990.

[Brungart '0I] D. Brungart, "Informational and energetic masking effects in the perception of two simultaneous talkers," JASA 109(3), March 200I.

[Brungart et al. 'OI] D. Brungart, B. Simpson, M. Ericson, K. Scott, "Informational and energetic masking effects in the perception of multiple simultaneous talkers," JASA I I0(5), Nov. 200 I.

[Brungart et al. '02] D. Brungart \& B. Simpson, “The effects of spatial separation in distance on the informational and energetic masking of a nearby speech signal”, JASA II 2(2), Aug. 2002.

[Brown \& Cooke '94] G. Brown \& M. Cooke,"Computational auditory scene analysis," Comp. Speech \& Lang. 8 (4), 297-336, 1994.

[Cooke et al. '0I] M. Cooke, P. Green, L. Josifovski, A.Vizinho,"Robust automatic speech recognition with missing and uncertain acoustic data," Speech Communication 34, 267-285, $200 \mathrm{I}$.

[Cooke’06] M. Cooke, “A glimpsing model of speech perception in noise,” submitted to JASA.

[Darwin \& Carlyon '95] C. Darwin \& R. Carlyon, "Auditory grouping” Handbk of Percep. \& Cogn. 6: Hearing, 387424, Academic Press, 1995.

[Ellis'96] D. Ellis, “Prediction-Driven Computational Auditory Scene Analysis,” Ph.D. thesis, MIT EECS, 1996.

[Hu \& Wang '04] G. Hu and D.L.Wang, "Monaural speech segregation based on pitch tracking and amplitude modulation," IEEE Tr. Neural Networks, I5(5), Sep. 2004.

[Okuno et al. '99] H. Okuno,T. Nakatani,T. Kawabata, “Listening to two simultaneous speeches," Speech Communication 27, 299-310, 1999. 


\section{References 2/2}

[Ozerov et al. '05] A. Ozerov, P. Phillippe, R. Gribonval, F. Bimbot, “One microphone singing voice separation using source-adapted models," Worksh. on Apps. of Sig. Proc. to Audio \& Acous., 2005.

[Pearlmutter \& Zador '04] B. Pearlmutter \& A. Zador, “Monaural Source Separation using Spectral Cues,” Proc. ICA, 2005.

[Parra \& Spence '00] L. Parra \& C. Spence, "Convolutive blind source separation of non-stationary sources," IEEE Tr. Speech \& Audio, 320-327, 2000.

[Reyes et al. '03] M. Reyes-Gómez, B. Raj, D. Ellis, "Multi-channel source separation by beamforming trained with factorial HMMs," Worksh. on Apps. of Sig. Proc. to Audio \& Acous., 13-16, 2003.

[Roman et al. '02] N. Roman, D.-L.Wang, G. Brown, “Location-based sound segregation,” ICASSP, I- I0I3-1016, 2002.

[Roweis '03] S. Roweis, "Factorial models and refiltering for speech separation and denoising," EuroSpeech, 2003.

[Schimmel \& Atlas '05] S. Schimmel \& L.Atlas, “Coherent Envelope Detection for Modulation Filtering of Speech," ICASSP, I-22I-224, 2005.

[Slaney \& Lyon '90] M. Slaney \& R. Lyon, “A Perceptual Pitch Detector,” ICASSP, 357-360, 1990.

[Smaragdis '98] P. Smaragdis, “Blind separation of convolved mixtures in the frequency domain,” Intl.Wkshp. on Indep. \& Artif.I Neural Networks, Tenerife, Feb. 1998.

[Seltzer et al. '02] M. Seltzer, B. Raj, R. Stern, "Speech recognizer-based microphone array processing for robust hands-free speech recognition,” ICASSP, I-897-900, 2002.

[Varga \& Moore '90] A.Varga \& R. Moore, "Hidden Markov Model decomposition of speech and noise," ICASSP, 845-848, 1990.

[Vincent et al. '06] E.Vincent, R. Gribonval, C. Févotte, “Performance measurement in Blind Audio Source Separation.” IEEE Trans. Speech \& Audio, in press.

[Yilmaz \& Rickard '04] O.Yilmaz \& S. Rickard, "Blind separation of speech mixtures via time-frequency masking," IEEE Tr. Sig. Proc. 52(7), I 830-I847, 2004. 